\title{
Enhanced Kerr nonlinearity and nonlinear figure of merit in silicon nanowires integrated with 2D graphene oxide films
}

\section{Supporting Information}

Yuning Zhang, ${ }^{\dagger}, \S$ Jiayang $W u,{ }^{\dagger}, \S$ Yunyi Yang, ${ }^{\dagger}+{ }^{\ddagger}$ Yang $Q u,{ }^{\dagger}$ Linnan Jia,${ }^{\dagger}$ Tania Moein,${ }^{\dagger}$

Baohua Jia, $*,+\ddagger$ and David J. Moss $* \dagger$

$\dagger$ Optical Sciences Centre, Swinburne University of Technology, Hawthorn, VIC 3122,

Australia

* Centre for Translational Atomaterial, Swinburne University of Technology, Hawthorn,

VIC 3122, Australia

*Email: bjia@,swin.edu.au,dmoss@swin.edu.au

Number of pages: 11

Number of figures: 7 
This Supporting Information comprises the following Sections:

I. Device fabrication

II. Experimental setup

III. Broadening factor

IV. Pulse energy incident at GO coated segments

V. Interplay between SPM and SA

VI. Modeling of nonlinear loss of silicon

VII. Fit SPM spectra

VIII. Influence of GO coated on sidewalls of SOI nanowires 


\section{Device fabrication}

Figure S1 shows the fabrication process flow for the GO-coated silicon nanowires on an SOI wafer with a 220 -nm-thick top silicon layer and a $2-\mu \mathrm{m}$-thick buried oxide (BOX) layer. First, silicon waveguides were fabricated by deep ultraviolet photolithography and inductively coupled plasma etching, with a $1.5-\mu \mathrm{m}$-thick silica being deposited by plasma enhanced chemical vapor deposition as an upper cladding layer. After that, windows with two different lengths of $0.4 \mathrm{~mm}$ and $2.2 \mathrm{~mm}$ were opened on the silica cladding and down to the BOX layer via photolithography and reactive ion etching. Alignment markers, prepared by metal lift-off after photolithography and electron beam evaporation, were used for accurate placement of the opened windows on the silicon nanowires. Finally, GO films were coated on the SOI nanowires by a solution-based method that yielded transfer-free, layer-by-layer GO film deposition. ${ }^{1,2}$

(i) SOI wafer

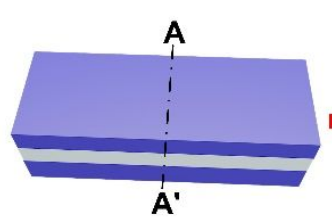

(ii) Waveguide patterning (iii) Cladding deposition $\&$ window opening

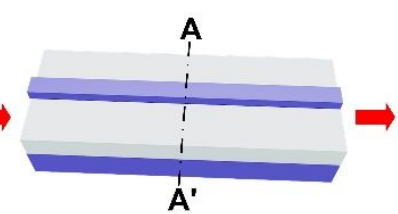

(iv) GO deposition
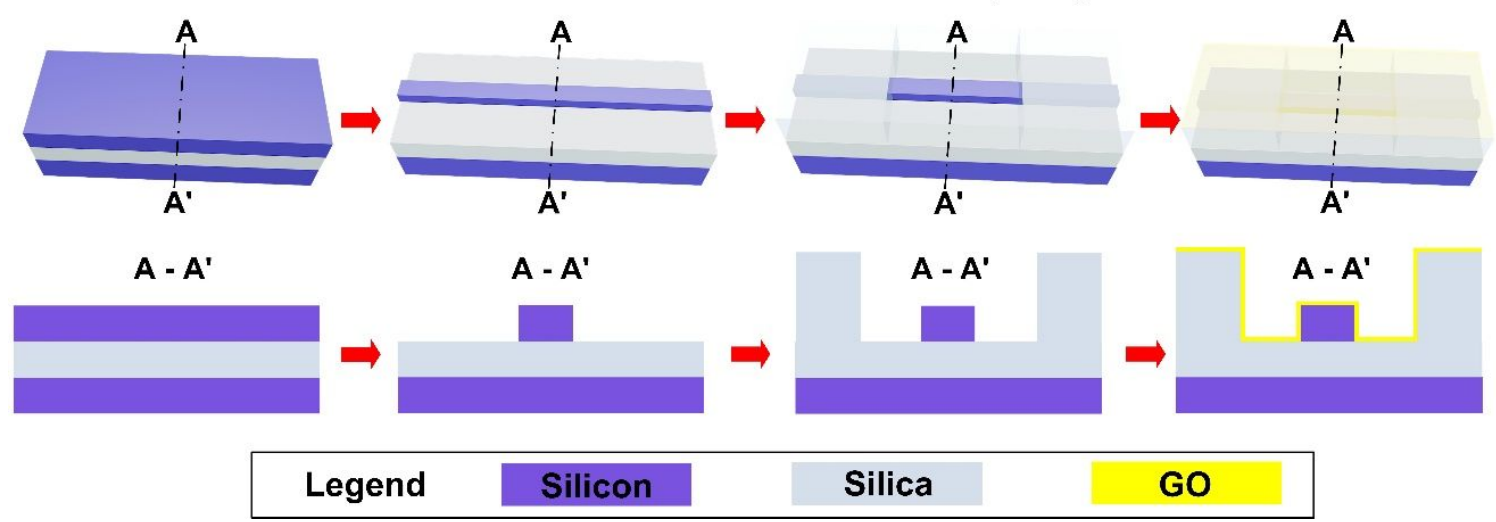

Figure S1. Schematic illustration showing the fabrication process flow. 


\section{Experimental setup}

\section{(i) Nonlinear loss measurement}

The nonlinear loss of the GO-coated SOI nanowires was measured using a pulsed fiber laser (PriTel, repetition rate: $\sim 60 \mathrm{MHz}$, pulse duration: $\sim 3.9 \mathrm{ps}$ ). The experimental setup is shown in Figure S2, where two optical power meters (OPM 1 and OPM 2) were employed to measure the average power of the optical pulses before and after transmission through the SOI nanowires. A variable optical attenuator (VOA) was used to tune the input pulse energy.

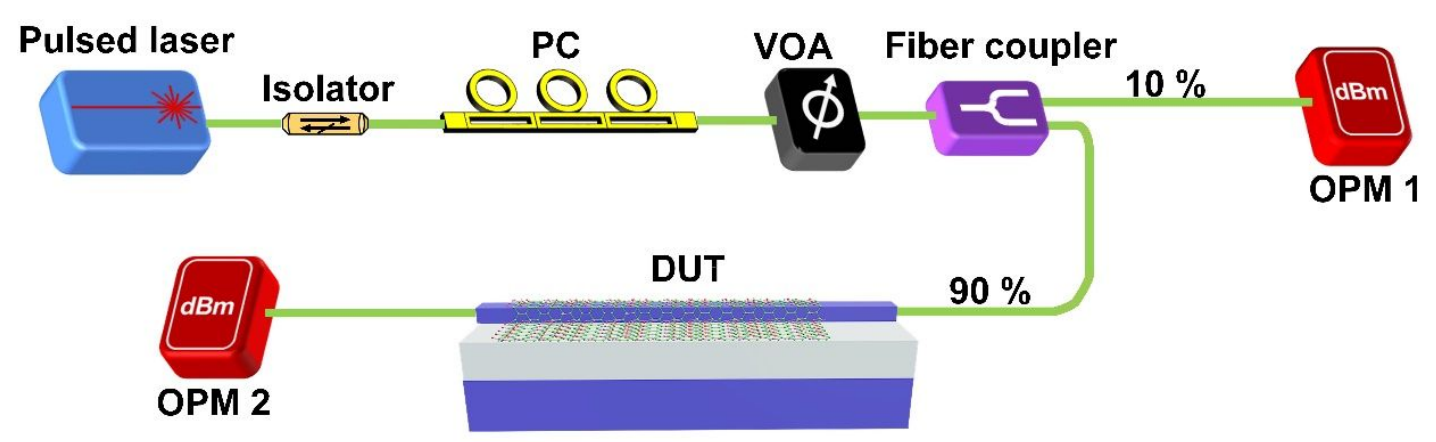

Figure S2. Experimental setup for measuring nonlinear loss of GO-coated SOI nanowires. PC: polarization controller. VOA: variable optical attenuator. OPM: optical power meter. DUT: device under test.

\section{(ii) SPM experiment}

We used the experimental setup shown in Figure S3 to perform SPM measurements on the GO-coated SOI nanowires. Picosecond optical pulses generated by a pulsed fiber laser (the same as the experimental setup for nonlinear loss measurement) were delivered into the hybrid waveguides, with a VOA to tune the input pulse energy. An optical isolator was inserted before the device under test (DUT) to prevent the reflected light from damaging the laser source and a polarization controller (PC) was used to set the input light to TE- 
polarization. The signal output from the DUT was split by a 90:10 coupler $-10 \%$ sent into an optical power meter (OPM) for power monitoring and the other $90 \%$ into an optical spectrum analyzer (OSA, Yokogawa AQ6370D) to observe the spectral broadening.

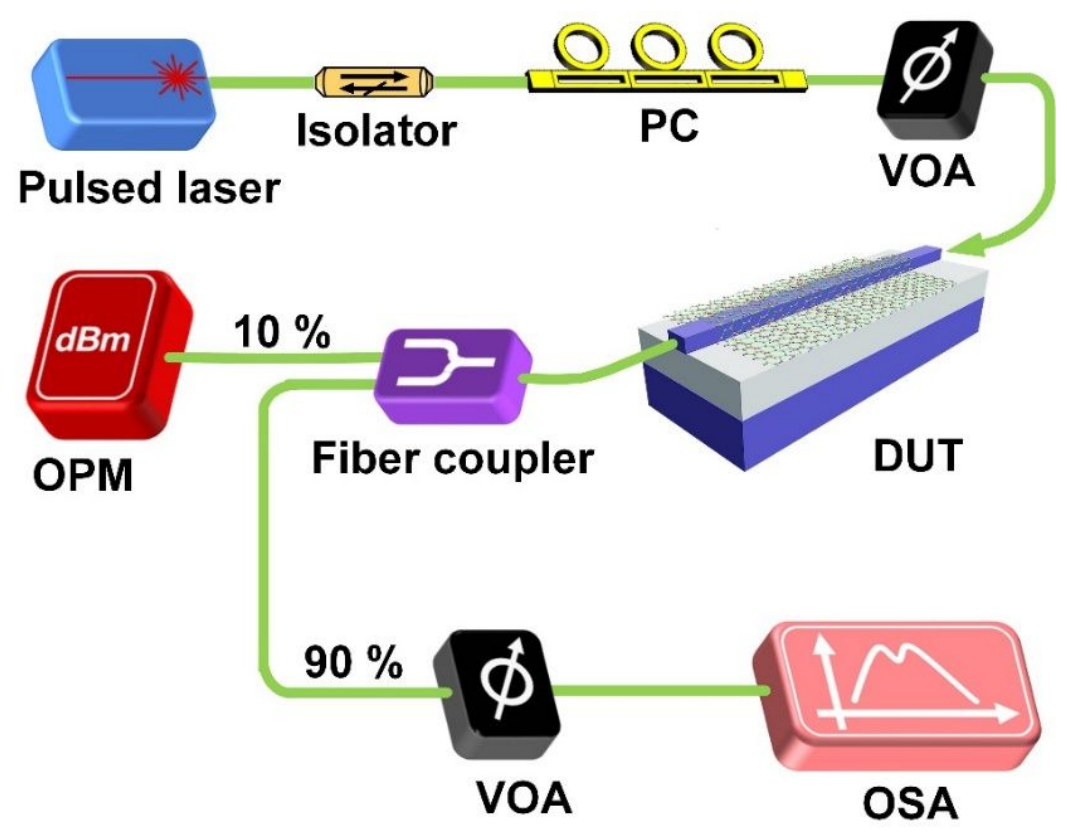

Figure S3. Experimental setup for SPM measurement in GO-coated SOI nanowires. PC: polarization controller. VOA: variable optical attenuator. OPM: optical power meter. DUT: device under test. OSA: optical spectrum analyzer.

\section{Broadening factor}

Broadening factor $(\mathrm{BF})$ is used for quantitively analyzing the spectral broadening of the output spectra due to SPM. The width of the optical spectra is described by the root-meansquare (RMS) width, defined as 3,4

$$
\Delta \omega_{r m s}^{2}=\left\langle\left(\omega-\omega_{0}\right)^{2}\right\rangle-\left\langle\left(\omega-\omega_{0}\right)\right\rangle^{2}
$$

where the angle brackets denote the average over the spectrum given by 


$$
\left\{\begin{array}{l}
\left\langle\left(\omega-\omega_{0}\right)^{2}\right\rangle=\frac{\int_{-\infty}^{\infty}\left(\omega-\omega_{0}\right)^{2} S(\omega) d \omega}{\int_{-\infty}^{\infty} S(\omega) d \omega} \\
\left\langle\left(\omega-\omega_{0}\right)\right\rangle^{2}=\left[\frac{\int_{-\infty}^{\infty}\left(\omega-\omega_{0}\right) S(\omega) d \omega}{\int_{-\infty}^{\infty} S(\omega) d \omega}\right]
\end{array}\right.
$$

where $S(\omega)$ is the spectrum intensity. The BF is therefore defined as:

$$
B F=\frac{\Delta \omega_{r m s}}{\Delta \omega_{0}}
$$

where $\Delta \omega_{0}$ is the RMS spectral width of the input optical pulses.

\section{Pulse energy incident at GO coated segments}

Figure $\mathrm{S} 4$ shows the pulse energy incident at the 2.2-mm-long and 0.4-mm-long GO coated segments as a function of the coupled pulse energy. We exclude the nonlinear loss induced by the SOI nanowires before the GO coated segments $(0.4 \mathrm{~mm}$ for the devices with 2.2mm-long GO films and $1.3 \mathrm{~mm}$ for the devices with 0.4-mm-long GO films) from the total pulse energy coupled into the hybrid waveguides and obtain the pulse energy at the start of the GO coated segments, which is the pulse energy for SA. 


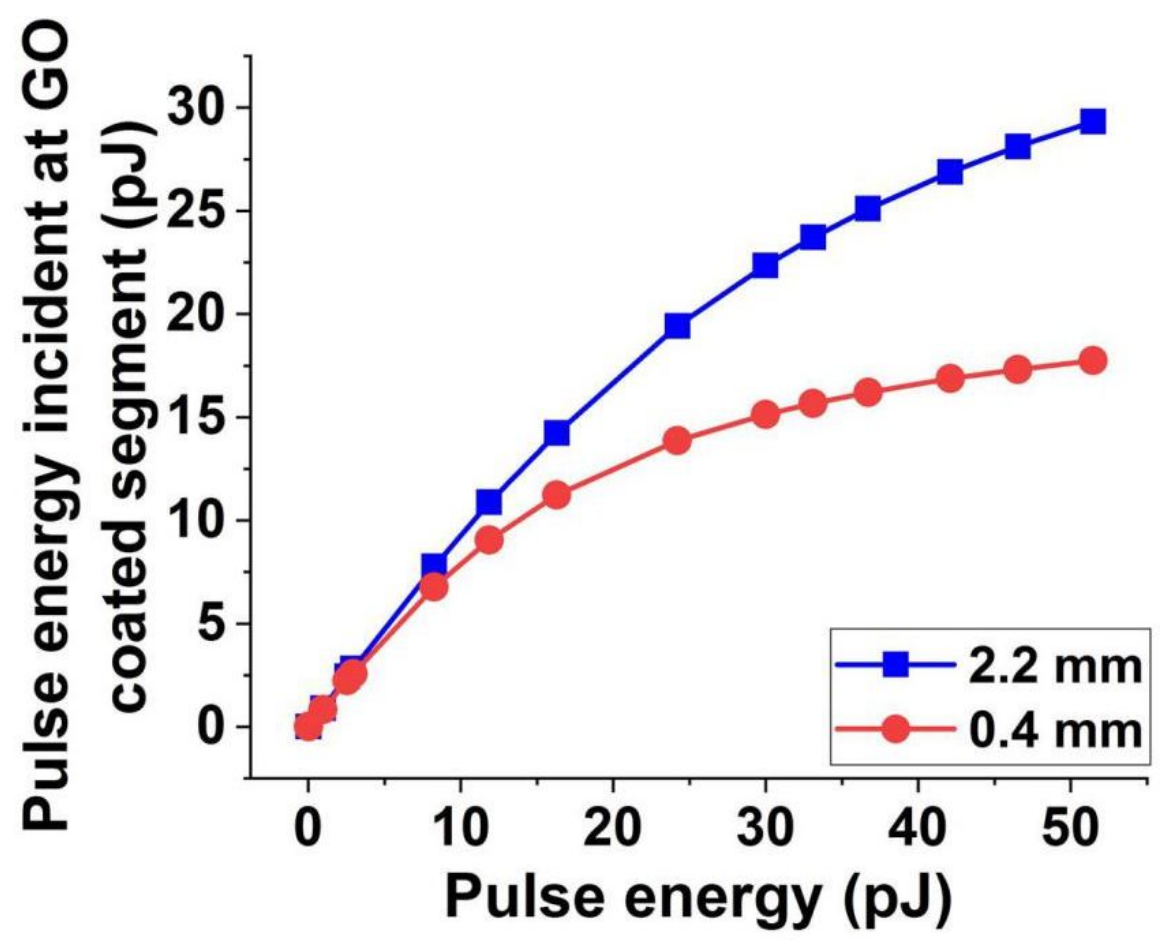

Figure S4. Pulse energy incident at the 2.2-mm-long and 0.4-mm-long GO coated segments versus pulse energy coupled into the hybrid waveguides.

\section{Interplay between SPM and SA}

Based on the SA loss modeling, we calculated $\triangle \mathrm{EIL}$ of the hybrid waveguides with and without considering spectral broadening induced by Kerr nonlinearity of GO. The results are shown in Table $\mathrm{S} 1$ for comparison. The absolute deviation $(A D)$ is calculated by

$$
A D=\left|\frac{\Delta E I L 2-\Delta E I L 1}{\Delta E I L 2}\right| \times 100 \%
$$

where $\triangle \mathrm{EIL} 1$ and $\triangle \mathrm{EIL} 2$ are the $\triangle \mathrm{EIL}$ of the hybrid waveguides with and without considering spectral broadening induced by Kerr nonlinearity of GO, respectively. $\triangle \mathrm{EIL1}$ and $\Delta$ EIL2 were calculated at the maximum coupled pulse energy of $\sim 51.5 \mathrm{pJ}$. The $n_{2}$ of $\mathrm{GO}$ is assumed to be zero when we calculated $\triangle \mathrm{EIL} 2$. In Table $\mathrm{S} 1$, the maximum difference induced by GO-induced spectral broadening on $\triangle \mathrm{EIL}$ is $<1 \%$. Therefore, we neglected 
any potential spectral/temporal coupling effects that could induce interplay between SA and SPM.

Table S1. Comparison of $\Delta$ EIL of the hybrid waveguides with and without considering spectral broadening induced by Kerr nonlinearity of GO. AD: absolute deviation.

\begin{tabular}{ccccc}
\hline $\begin{array}{c}\text { GO coating } \\
\text { length }\end{array}$ & $\begin{array}{c}\text { GO layer } \\
\text { number }\end{array}$ & $\Delta \mathrm{EIL} 1(\mathrm{~dB})$ & $\Delta \mathrm{EIL} 2(\mathrm{~dB})$ & $\mathrm{AD}$ \\
\hline \multirow{3}{*}{$2.2 \mathrm{~mm}$} & 1 & -0.60837 & -0.6107 & $0.38 \%$ \\
& 2 & -1.00634 & -1.014 & $0.75 \%$ \\
& 3 & -1.5739 & -1.5873 & $0.84 \%$ \\
\hline \multirow{3}{*}{$0.4 \mathrm{~mm}$} & 5 & -0.5688 & -0.5689 & $0.017 \%$ \\
& 10 & -1.1494 & -1.1496 & $0.017 \%$ \\
& 20 & -1.9662 & -1.9543 & $0.6 \%$ \\
\hline
\end{tabular}

\section{Modeling of nonlinear loss of silicon}

The $\alpha_{N L-S O I}$ includes the nonlinear losses induced by TPA and FCA of silicon, which are: ${ }^{5}$

$$
\alpha_{N L-S O I}=\frac{\beta_{T P A, S i}}{A_{e f f}}|A|^{2}+\sigma N_{c}
$$

where $\beta_{T P A, S i}$ is the TPA coefficient of silicon and $A_{\text {eff }}$ is the effective mode area of the waveguides. $N_{c}$ results from free carriers generated in silicon, given by: 5

$$
\frac{\partial N_{\mathrm{c}}(z, t)}{\partial t}=\frac{\beta_{T P A, S i}}{2 \hbar_{\omega}} \cdot \frac{|A(z, t)|^{4}}{A_{e f f}{ }^{2}}-\frac{N_{c}(z, t)}{\tau_{c}}
$$

where $\hbar$ is the Planck's constant, $\omega$ is the angular frequency, $\tau_{c}=\sim 1 \mathrm{~ns}$ is the effective carrier lifetime. The $\tau_{c}$ term in Eq. (S6) can be ignored since the pulse duration $(\sim 3.9 \mathrm{ps})$ is much less than $\tau_{c}$ so that the generated free carriers do not have time to recombine within the pulse duration..$^{5,6}$ The measured EIL of the bare SOI nanowire as a function of the 
coupled pulse energy is shown in Figure S5 along with the fit EIL calculated from Eqs. (S5)-(S6). The fit $\beta_{T P A, S i}$ and $\sigma$ are $5.02 \times 10^{-12} \mathrm{~m} / \mathrm{W}$ and $1.44 \times 10^{-21} \mathrm{~m}^{2}$, respectively, which agree well with the literature, ${ }^{5}$ confirming that the nonlinear loss of the bare SOI nanowire was mainly induced by TPA and FCA. We also note that the free-carrier effect of silicon including both FCA and FCD significantly affected the output spectral shapes, largely accounting for the significant asymmetry.

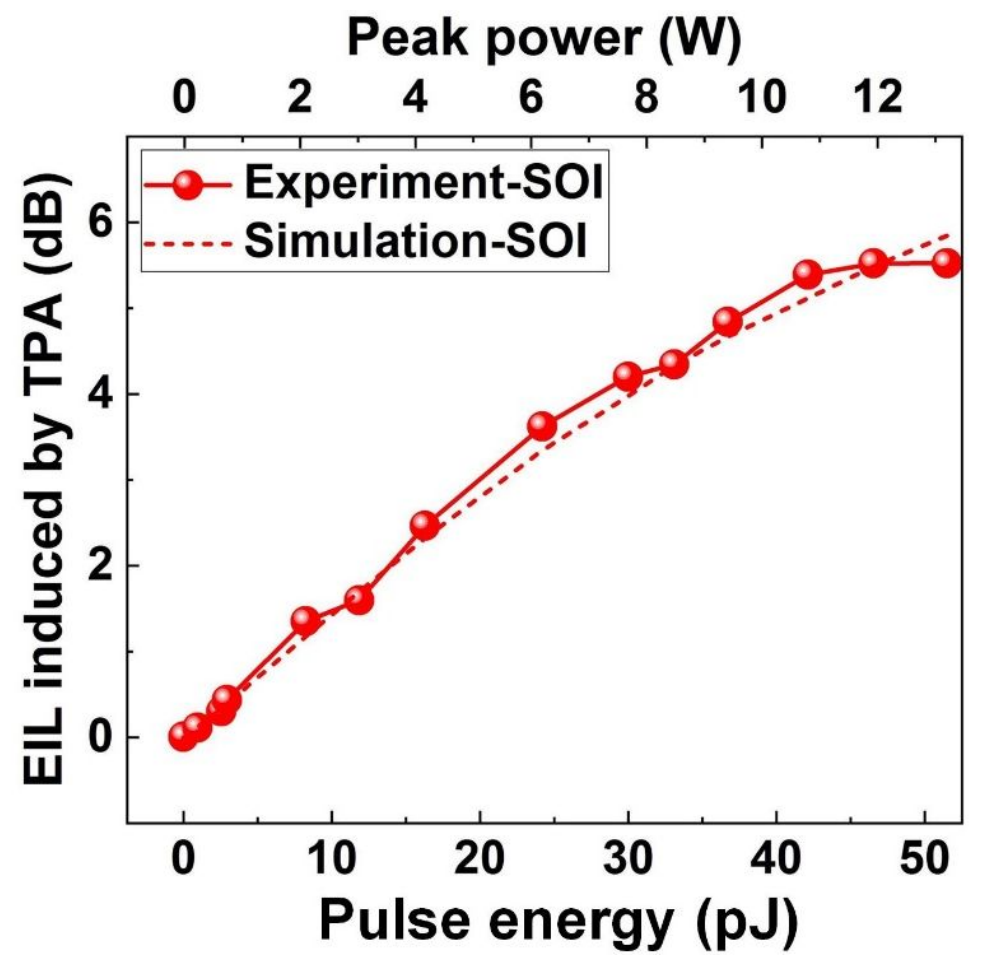

Figure S5. Measured and simulated EIL induced by TPA and FCA of silicon.

\section{Fit SPM spectra}

Based on the SPM modeling, we fit the experimentally measured spectra to obtain the effective nonlinear parameter $\gamma_{e f f}$ of the hybrid waveguides. Figure S6a shows the measured and fit optical spectra for the input pulses. We approximated the input pulse shape by a Gaussian profile as below: 


$$
A=\sqrt{P_{0}} \cdot \exp \left[-\frac{1}{2}\left(1+i C_{0}\right)\left(\frac{t}{T_{0}}\right)^{2}\right]
$$

where $P_{0}$ is the pulse peak power, $C_{0}$ is the initial chirp, and $T_{0}$ is se duration. The measured and fit optical spectra for the output signal after transmission through the bare SOI nanowire at a coupled pulse energy of $51.5 \mathrm{pJ}$ are shown in Figure S6b, showing good agreement between theory and experiment, with the discrepancy arising mainly from small imperfections in the input pulse spectrum. We obtain a $\gamma$ of $288 \mathrm{~W}^{-1} \mathrm{~m}^{-1}$ for the bare SOI nanowire, in agreement with previous reports. ${ }^{7}$ The measured and fit optical spectra for the output signals after transmission through the SOI nanowires with 2 and 10 layers of GO are shown in Figures S6c and d, respectively, where the coupled pulse energy is the same as Figure S6b. Here again, we obtain good agreement between theory and experiment. The absolute deviation between the BFs of the measured and fit spectra is depicted in Figure S6e, which is mainly resulted from the slight difference between the measured and fit output spectra. As can be seen, the BFs of the fit spectra closely match with the BFs of the measured spectra, with the absolute deviation $<3.2 \%$. 

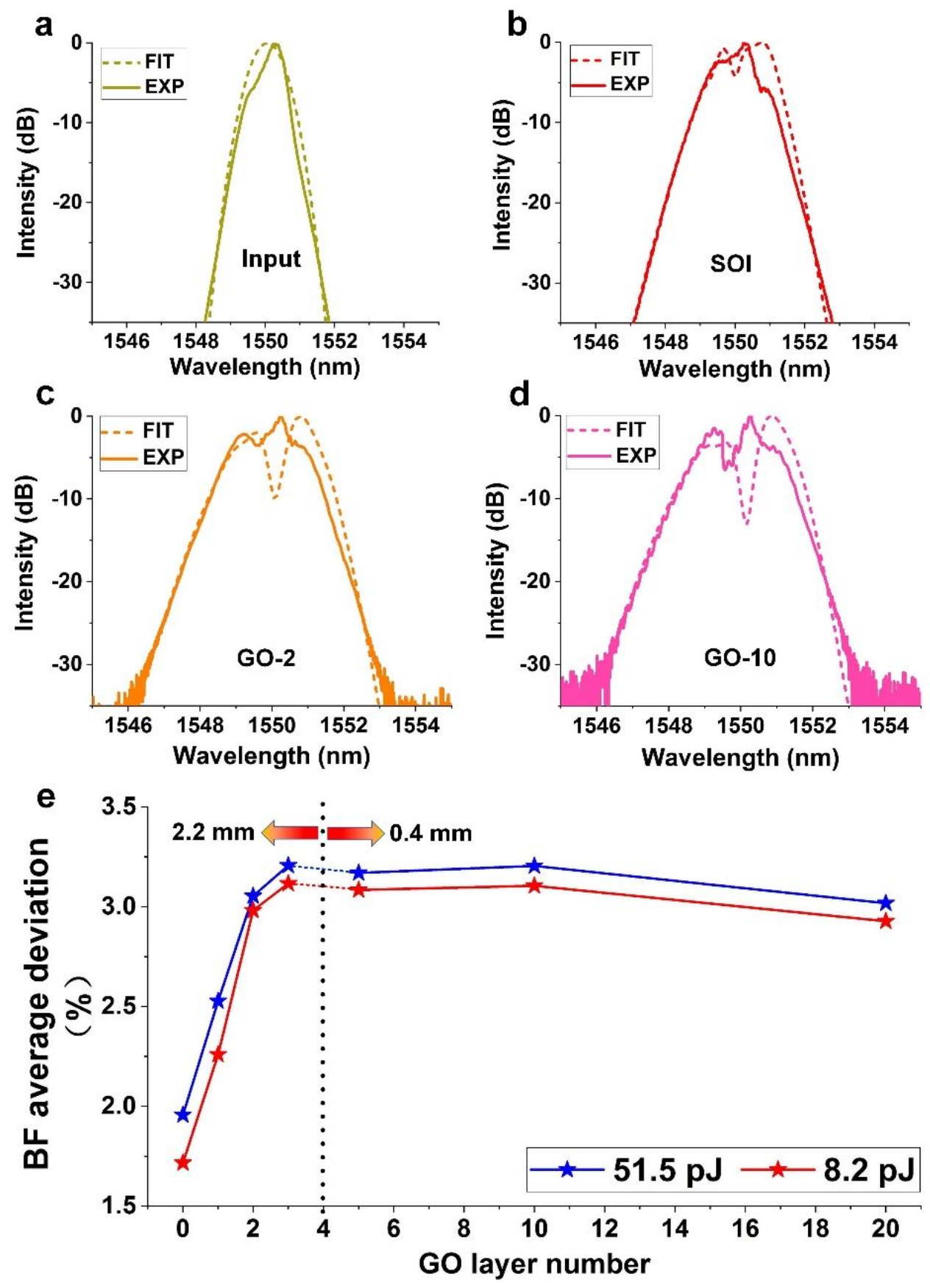

Figure S6. (a)-(d) Measured and fit optical spectra for (a) input optical pulses, (b) output signal after transmission through the bare SOI nanowire, (c) output signal after transmission through the SOI nanowire with 2.2-mm-long, 2 layers of GO, and (d) output signal after transmission through the SOI nanowire with 0.4-mm-long, 10 layers of GO. The coupled pulse energy in (b)-(d) is $\sim 51.5$ pJ. (e) Average deviation between the BFs of the measured and fit spectra at coupled pulse energies of $8.2 \mathrm{pJ}$ and $51.5 \mathrm{pJ}$. The layer number ' 0 ' corresponds to the results for the bare SOI nanowire. 


\section{Influence of GO coated on sidewalls of SOI nanowires}

Our GO film coating method enables conformal coating of GO films on SOI nanowires, with GO films being coated on both the top surface and sidewalls of the waveguides. This is slightly different to the doped silica devices with GO films only coated on the top surface of the waveguides. ${ }^{8,9,10}$ Figure $\mathrm{S} 7$ shows the ratio of the power in GO on both sidewalls to that in all of the GO material regions caculated by FDTD simulations. The ratio is $<3 \%$ for all devices (i.e., all numbers of GO layers that we used), indicating that the TE mode overlap with GO on the sidewalls was negligible compared with the top of the nanowires. We therefore neglected any possible difference between the in-plane and out-of-plane $n_{2}$ of the layered GO films.

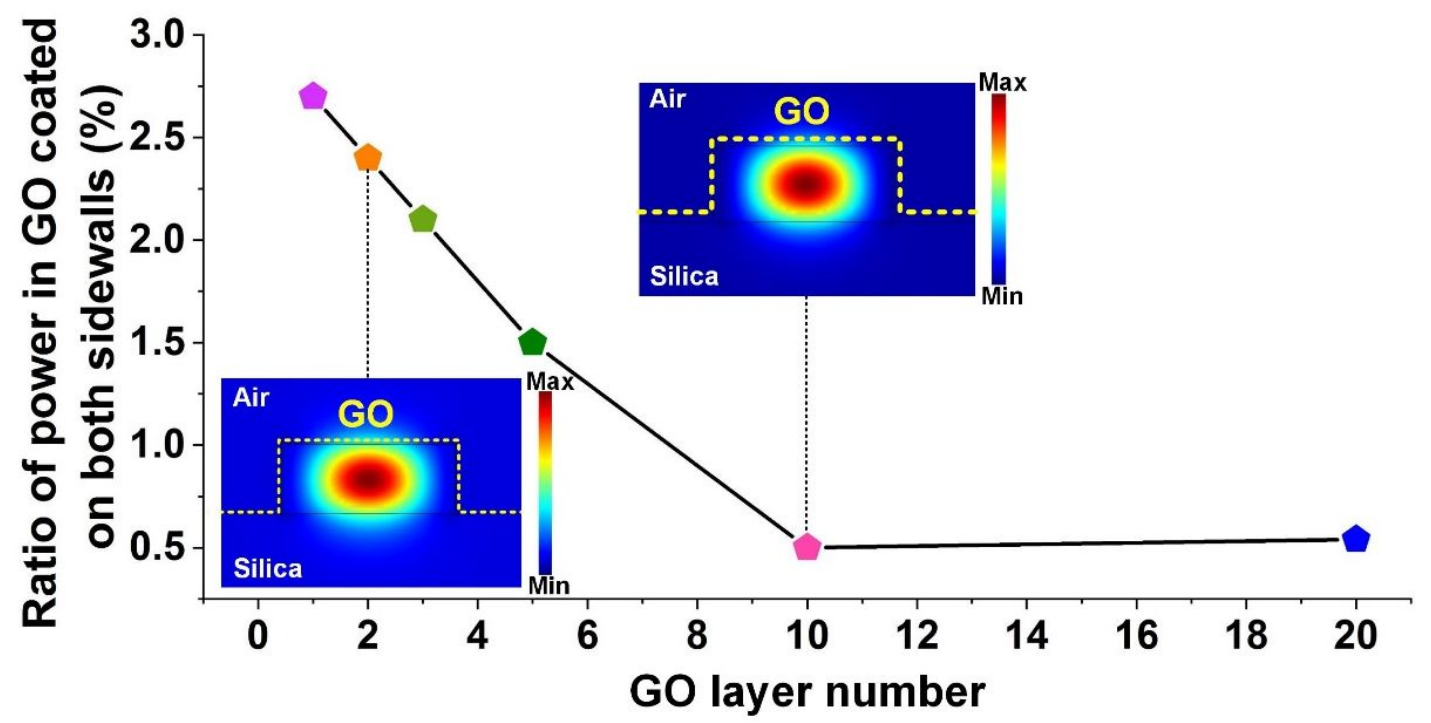

Figure S7. Ratio of power in GO coated on both sidewalls to that in all GO material regions. Insets show the TE mode profiles for the SOI nanowires with 2 and 10 layers of GO, respectively. 


\section{REFERENCES}

1. Yang, Y.; Wu, J.; Xu, X.; Liang, Y.; Chu, S. T.; Little, B. E.; Morandotti, R.; Jia, B.; Moss, D. J., Invited Article: Enhanced Four-Wave Mixing in Waveguides Integrated with Graphene Oxide. APL Photonics 2018, $3(12), 120803$.

2. Yang, Y. Y.; Lin, H.; Zhang, B. Y.; Zhang, Y. N.; Zheng, X. R.; Yu, A. M.; Hong, M. H.; Jia, B. H., Graphene-Based Multilayered Metamaterials with Phototunable Architecture for on-Chip Photonic Devices. ACS Photonics 2019, 6 (4), 1033-1040.

3. Vermeulen, N.; Castello-Lurbe, D.; Khoder, M.; Pasternak, I.; Krajewska, A.; Ciuk, T.; Strupinski, W.;

Cheng, J.; Thienpont, H.; Van Erps, J., Graphene's Nonlinear-Optical Physics Revealed Through

Exponentially Growing Self-Phase Modulation. Nat Commun 2018, 9 (1), 2675

4. Demongodin, P.; El Dirani, H.; Lhuillier, J.; Crochemore, R.; Kemiche, M.; Wood, T.; Callard, S.; RojoRomeo, P.; Sciancalepore, C.; Grillet, C.; Monat, C., Ultrafast Saturable Absorption Dynamics in Hybrid Graphene/ $\mathrm{Si}_{3} \mathrm{~N}_{4}$ Waveguides. APL Photonics 2019, 4 (7), 076102.

5. Yin, L. H.; Agrawal, G. P., Impact of Two-Photon Absorption on Self-Phase Modulation in Silicon Waveguides. Opt Lett 2007, 32 (14), 2031-2033.

6. Feng, Q.; Cong, H.; Zhang, B.; Wei, W.; Liang, Y.; Fang, S.; Wang, T.; Zhang, J., Enhanced Optical Kerr Nonlinearity of Graphene/Si Hybrid Waveguide. Appl Phys Lett 2019, 114 (7), 071104.

7. Leuthold, J.; Koos, C.; Freude, W., Nonlinear silicon photonics. Nat Photonics 2010, 4 (8), 535-544.

8. Yang, Y.; Wu, J.; Xu, X.; Liang, Y.; Chu, S. T.; Little, B. E.; Morandotti, R.; Jia, B.; Moss, D. J., Invited Article: Enhanced Four-Wave Mixing in Waveguides Integrated with Graphene Oxide. APL Photonics 2018, $3(12), 120803$.

9. Wu, J.; Yang, Y.; Qu, Y.; Xu, X.; Liang, Y.; Chu, S. T.; Little, B. E.; Morandotti, R.; Jia, B.; Moss, D. J., Graphene Oxide Waveguide and Micro - Ring Resonator Polarizers. Laser Photonics Rev 2019, 1900056.

10. Wu, J.; Yang, Y.; Qu, Y.; Jia, L.; Zhang, Y.; Xu, X.; Chu, S. T.; Little, B. E.; Morandotti, R.; Jia, B.; Moss, D. J., 2D Layered Graphene Oxide Films Integrated with Micro-Ring Resonators for Enhanced Nonlinear Optics. Small 2020, e1906563. 